\title{
Comparative Study of DCT based Image Compression on Hexagonal and Conventional Square Pixel Images
}

\author{
Jeevan K.M. \\ Dept. of Electronics and Communication, \\ Sreenarayana Gurukulam College of Engineering, \\ Ernakulam,India
}

\begin{abstract}
Even though, the storage space and transmission bandwidth can be reduced by compression, it will reduce the image fidelity, especially when the images are compressed at lower bit rates. Discrete Cosine Transform (DCT) based compression is an effective way of compression in which good compression ratio without losing too much of information can be obtained. In this work a comparison of DCT based Image compression on hexagonal and rectangular sampling grid is performed. Mean Square Error and Peak Signal to Noise Ratio is considered for the performance analysis. Compression on hexagonal domain gives better results compared to compression on rectangular domain
\end{abstract}

\section{General Terms}

Image compression, DCT, Image Processing

\section{Keywords}

Image resampling, Hexagonal Image, Alternate pixel.

\section{INTRODUCTION}

The conventional representation for digital images is using a square shaped picture element known as pixels but in hexagonal grid image, instead of square shaped pixel we are considering the shape of hexagon for the basic picture element. Image processing in hexagonal grid is very much advantageous than in the conventional rectangular grid. The idea behind using a hexagonal pixel grid model is that retina of the eye closely resembles a hexagonal grid space. If we will be able to model and process the images on such a grid space, one can imitate natural behavior to realize Computer Vision. Higher angular resolution, consistent connectivity, higher sampling efficiency, smaller quantization error, equidistance and higher Symmetry are some of the advantage of processing images in hexagonal domain [1]. Due to the non availability of hardware for capturing and displaying hexagonal based images, it limits the use of hexagonal image structure for further processing. Since there is no hardware for capturing the hexagonal images, conversion has to be done from square to hexagonal image before hexagonal-based image processing.

Resampling is the process of transforming a discrete image which is defined at one set of coordinate locations to a new set of coordinate points i.e., converting from rectangular to hexagonal grid. The Fig.1 shows the system diagram for performing compression on hexagonal sampling grid.
In this work I proposed a hybrid approach to Image Compression. DCT based image compression is being applied to hexagonally converted images and hexagonal domain gives better results compared to rectangular domain.

\section{COMPRESSION OF HEXAGONAL IMAGE USING DCT}

Discrete Cosine Transform (DCT) based compression is an effective way of compression in which good compression ratio without losing too much of information can be obtained. The Discrete Cosine Transform (DCT) transforms the time domain input into a linear combination of weighted basis functions. These basis functions are commonly the frequency components of the input. Since image is two dimensional we use 2-D Discrete Cosine Transform, which is just a one dimensional DCT applied twice, once in the ' $x$ ' direction, and again in the ' $y$ ' direction. The DCT equation, computes the $i^{\text {th }}$ and $j^{\text {th }}$ entry of the DCT of an image[2].

$$
\begin{gathered}
F(k, l)=\alpha(k) \alpha(l) \sum_{m=0}^{N-1} \sum_{n=0}^{N-1} f(m, n) \cos \left[\frac{(2 m+1) \pi k}{2 N}\right], 0 \leq \leq N-1 \\
\alpha(k) \& \alpha(l)=\left\{\begin{array}{l}
\sqrt{\frac{1}{N}} \text { if } k, l=0 \\
\sqrt{\frac{2}{N}} \text { if } k, l \neq 0
\end{array}\right.
\end{gathered}
$$

Following are the steps involved in DCT based image compression [3].

(1).The image is broken into $8 \times 8$ blocks of pixels. (2). Working from left to right, top to bottom, the DCT is applied to each block. (3).Each block is compressed through quantization. (4).The array of compressed blocks that constitute the image is stored in reduced amount of space. 


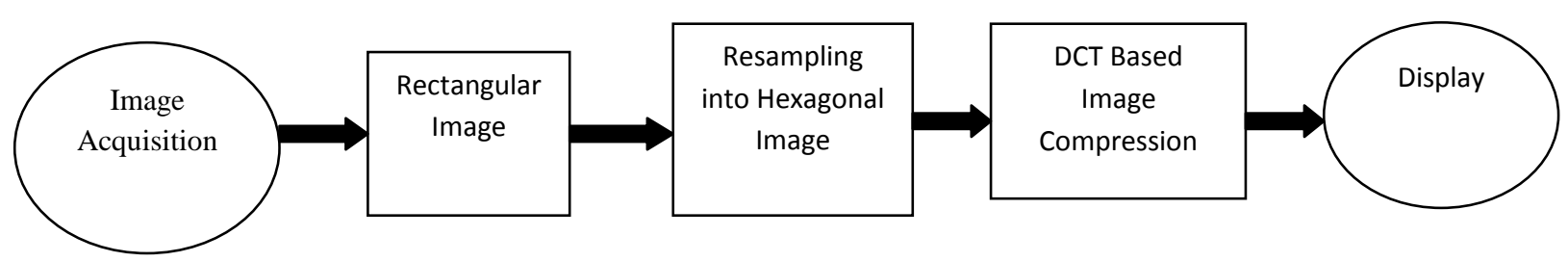

Fig 1: The system diagram for performing compression on hexagonal sampling grid

In this work DCT based compression is performed on hexagonal grid representation, which is based on alternate pixel suppressal method. Hexagonal grid image based on alternate pixel suppressal method can be obtained from the conventional image by alternatively suppressing rows and columns of the existing rectangular grid and sub sampling it [4]. All the other pixels of the rectangular grid which do not have any correspondence with the hexagonal counterparts are suppressed to zero. While processing this sub sampled image the suppressed pixels are not considered in computation. The sub sampled hexagonal grid using alternate pixel suppressal method is shown in Fig. 2

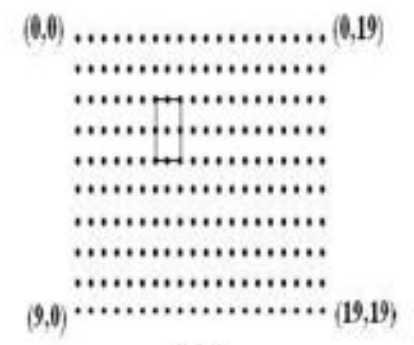

(a)

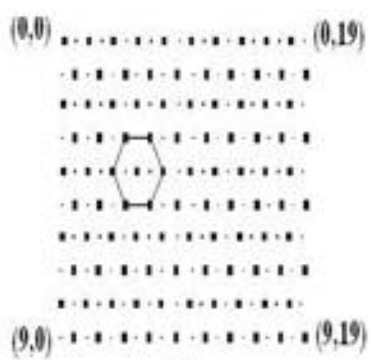

(b)
Fig 2: (a) Rectangular grid and (b) Simulated grid obtained using alternate pixel submission

\section{PERFORMANCE COMPARISON}

In this work, the performances of image compression on conventional square pixel image and hexagonal image are compared. The performance was compared based on the parameters, Compression Ratio (CR), Mean Square Error (MSE) and Peak Signal to Noise Ratio (PSNR).

If $\mathrm{n} 1$ and $\mathrm{n} 2$ are the number of information carrying units (no. of bits ) in two data sets that represent the same image, then the compression ratio is defined as $\mathrm{CR}=\mathrm{n} 1 / \mathrm{n} 2$.

MSE indicate the average difference of the pixel throughout the image. If MSE is higher the difference of the pixel between the original and the processed image is also higher. The equation for MSE is shown below

$$
M S E=\frac{1}{m n} \sum_{i=0}^{m-1} \sum_{j=0}^{n-1}[I(i, j)-K(i, j)]^{2}
$$

Where ' $\mathbf{m}$ ' and ' $\mathbf{n}$ ' are the row and column size of the image and $\mathrm{I}$ and $\mathrm{K}$ are the processed and the original image respectively.

$$
P S N R=10 \log _{10}\left(\frac{M A X_{i}^{2}}{M S E}\right)
$$

Where $\mathrm{MAX}_{\mathrm{I}}$ is the maximum possible pixel value of the image. When the pixels are represented using 8 bits per sample, this is 255 .

\section{RESULTS AND DISCUSSION}

DCT based compression of image on rectangular and hexagonal grid are performed and it is compared based on Compression Ratio, MSE and PSNR. The results obtained is shown in Table. 1

Table 1. Test results Comparison

\begin{tabular}{|l|l|l|l|l|}
\hline $\begin{array}{l}\text { Test } \\
\text { Images }\end{array}$ & Sampling & MSE & PSNR & CR \\
\hline \multirow{3}{*}{ water.gif } & Rectangular & 81.29 & 29.03 & 22.87 \\
\cline { 2 - 5 } & Hexagonal & 191.30 & 25.31 & 30.46 \\
\hline \multirow{2}{*}{ lena.gif } & Rectangular & 60.74 & 30.30 & 30.46 \\
\cline { 2 - 5 } & Hexagonal & 207.5 & 24.97 & 34.49 \\
\hline
\end{tabular}

The results show that, DCT based compression on hexagonal grid image produces better results than on rectangular grid image. The comparison shows that, hexagonal grid gives better PSNR and also reduced MSE for hexagonal grid. Fig. 3 and Fig. 4 show the original and the compressed images in rectangular and hexagonal domain for test images water.gif.

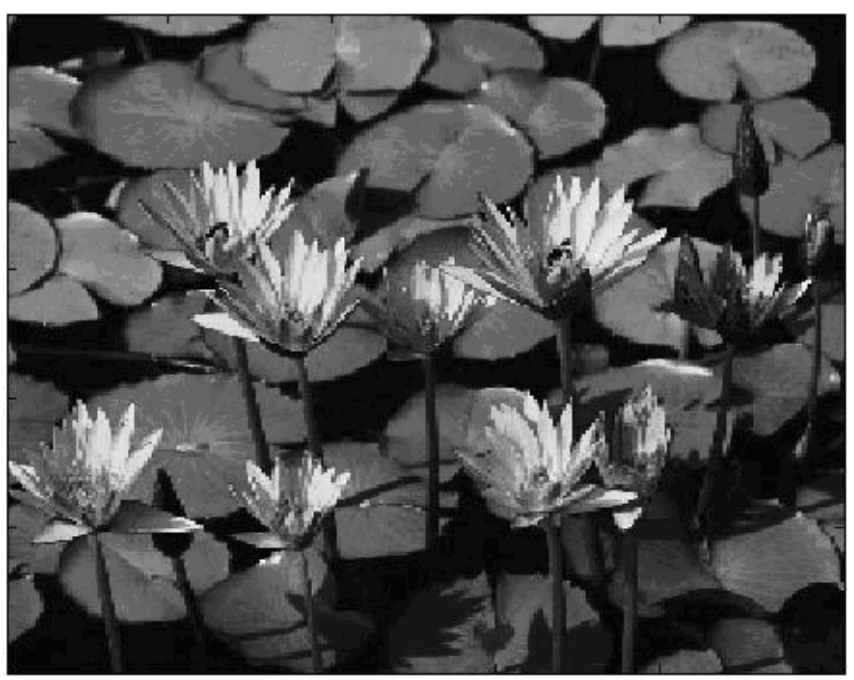

Fig 3: (a) Original Image (water.gif) 


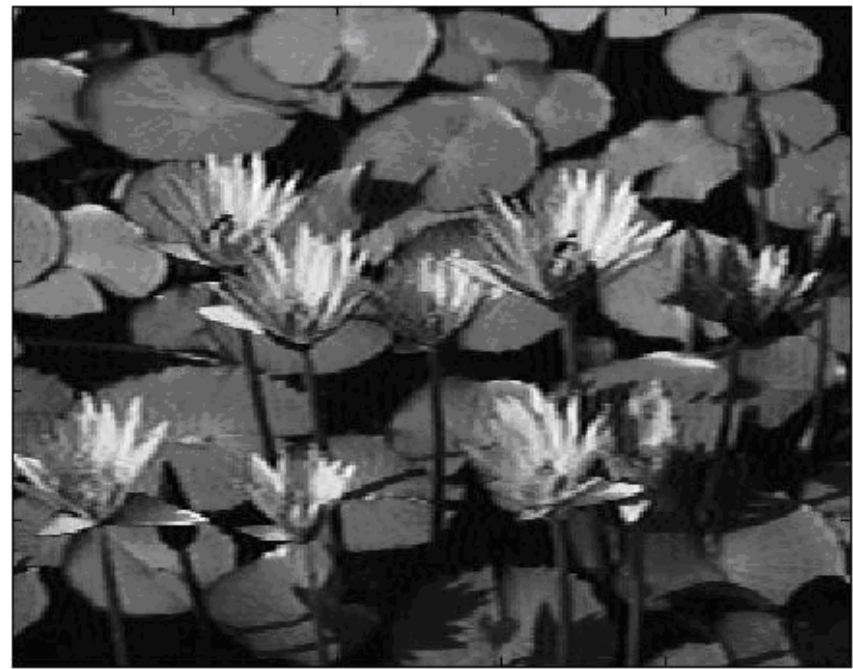

Fig 3: (b) Compressed Image (water.gif)

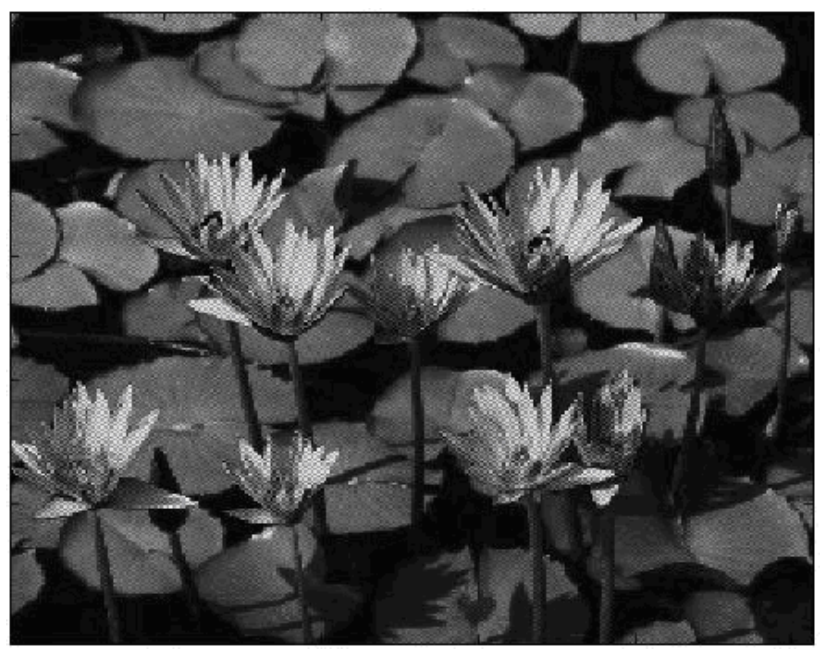

Fig 4: (a) Hexagonally resampled image (water.gif) based on alternate pixel suppressal method

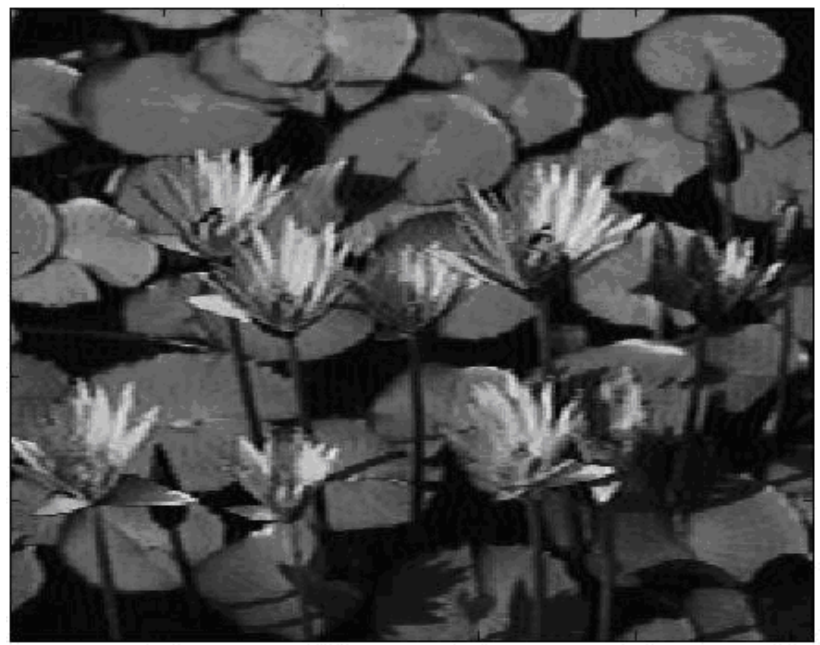

Fig 4: (b) Compressed version of Hexagonally resampled image (water.gif) based on alternate pixel suppressal method

Fig. 5 and Fig. 6 show the plot of CR v/s MSE and CR v/s PSNR respectively. This graph also shows that the hexagonal image processing gives better results for DCT based compression. In the case of conventional square pixel image as CR increases the MSE increases and the PSNR decreases. But in the case of Hexagonal image we are getting almost constant results.

\section{CONCLUSION}

In this work DCT based image compression is performed on both rectangular grid and hexagonal grid images. The performance is studied using Compression Ratio (CR),MSE and PSNR. Performance comparison shows better results for DCT based image compression in hexagonal grid images. Also from the graph we can see that in the case of hexagonal image, as the CR increases the PSNR does not decreases much, where as in conventional square pixel images PSNR goes down as CR increases. So better results will be obtained by using hexagonal images for DCT based compression.

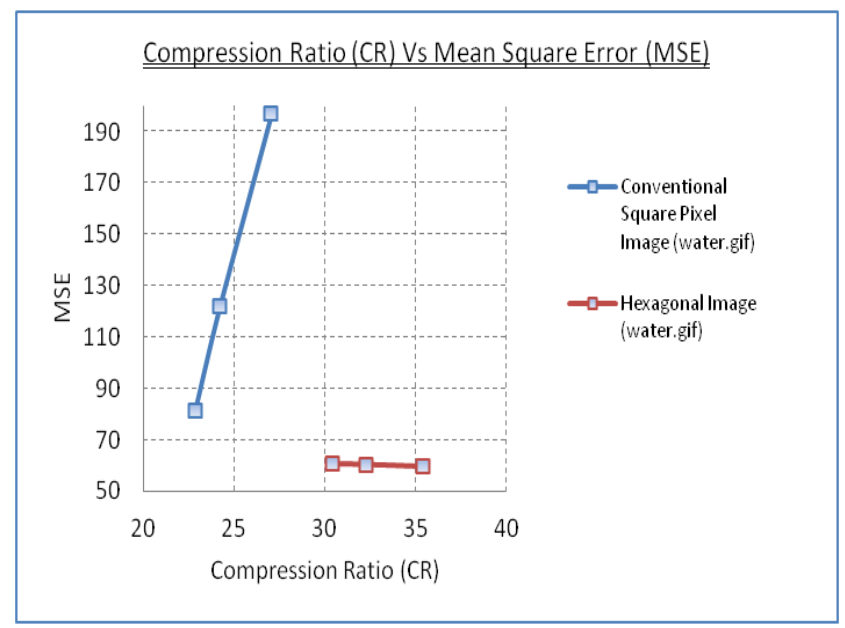

Fig 5: Graph of CR v/s MSE for the image water.gif

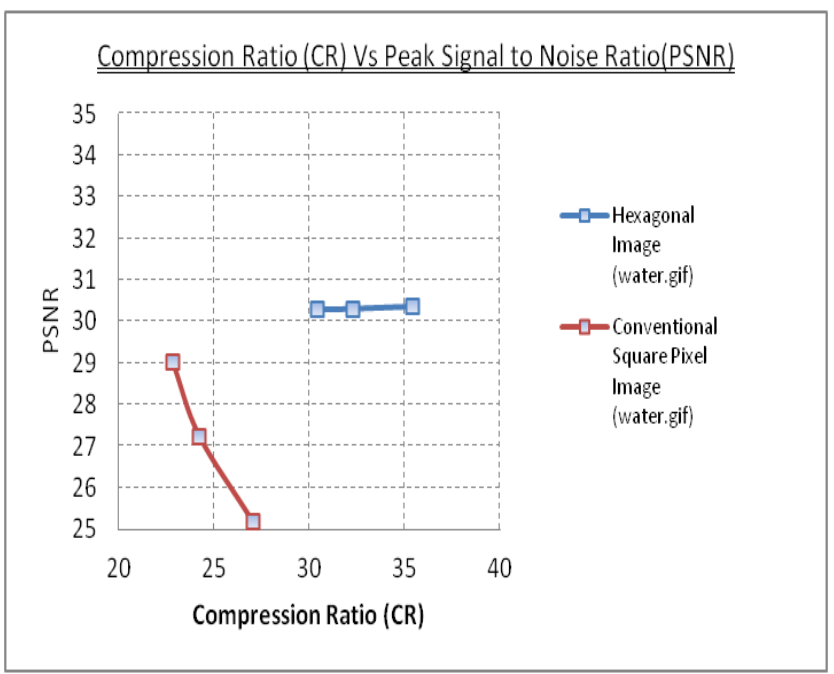

Fig 6: Graph of CR v/s PSNR for the image water.gif

\section{REFERENCES}

[1] K.P.Soman and K.I.Ramachandran, "Insight into wavelets from theory to practice", Second Edition, PHI, 2005

[2] Anil K. Jain. "Fundamentals of Digital Image Processing", Prentice Hall, 2001 
[3] Gonzalez, R.C. and R.E.Woods, "Digital image processing", New Jersey: Prentice Hall, 2002

[4] P.Vidya,S.Veni and K.A. Narayanankutty, "Performance Analysis of Edge Detection Methods on Hexagonal Sampling Grid", International Journal of Electronic Engineering Research, ISSN 0975 - 6450 Volume 1 Number 4 (2009) pp. 313-328

[5] P.Vidya, "Gabor Wavelet Based Edge Detection on Hexagonal Sampled Grid", The International Journal on Electronics \& Communication Technology, ISSN 22307109, Volume 2, Issue 2, June 2011, pp 122-125

[6] Staunton, R.C., "An Analysis of Hexagonal "Thinning Algorithms and Skeletal Shape Representation". Pattern Recognition, 29(7): p.1131-1146, 1996.

[7] Staunton, R.C., "A one pass parallel hexagonal thinning algorithm. Image Processing and Its Applications", 1999. Seventh International Conference on (Conf. Publ. No. 465), 2: p.841-845 vol.2, 1999
[8] Golay, M., "Hexagonal parallel pattern transformation". IEEE Transactions on computers, 18(8): p. 733-740, 1969.

[9] A.Laine,S.Schuler,"Hexagonal wavelet processing of digital mammography",Medical Imaging 1993, Part of SPIE's Thematic Applied Science and Engineering series, Newport Beach, California, February 14-19,1993.

[10] Qiang Wu, Xiangjian He and Tom Hintz, "Preliminary Image Compression Research Using Uniform Image Partitioning on Spiral Architecture", Proceedings of the 2nd International Conference on Information Technology for Application (ICITA 2004)

[11] E. Schwartz, "Computational Anatomy and Functional Architecture of Striate Cortex: A Spatial Mapping Approach to Perceptual Coding" Vision Research 20, pp.

[12] Mersereau, R.M., "The processing of Hexagonally Sampled Two-Dimensional Signals'. Proceedings of the IEEE, 67: p. 930-949,1979. 